\title{
Custeio-Alvo Incorporando o Custo de Capital para Tomada de Decisão: Um caso de Ensino
}

João Carlos Hipólito Bernardes do Nascimento Doutorado em Ciências Contábeis pela Universidade Federal do Rio de Janeiro - UFRJ

Professor na Universidade Federal do Piauí - UFPI Campus Universitário Ministro Petrônio Portella, s/n. Ininga. Teresina/PI. CEP: 64.049-

E-mail: joaohipolito@ufpi.edu.br

Washington Juarez de Brito Filho Doutorado em Direito Tributário pela Universidade de São Paulo - USP Professor na Universidade Federal do Rio de Janeiro - UFRJ Av. Pedro Calmon, 550. Cidade Universitária. Rio de Janeiro/RJ. CEP: 21.941-901

E-mail:wwwbrito@gmail.com

Juliana Reis Bernardes Mestrado em Administração e Desenvolvimento Empresarial pela Universidade Estácio de Sá Professora na Faculdade de Ensino Superior de Floriano - FAESF Rua Olemar Alves de Sousa, 401. Rede Nova. Floriano. Piauí. CEP: 64.809-170 E-mail: jureis3d@hotmail.com

Josaias Santana dos Santos Mestrado em Ciências Contábeis pela Fucape Bussines School - FUCAPE Servidor Público Federal. Contador da Universidade Federal do Vale do São Francisco - UNIVASF Av. José de Sá Maniçoba, S/N. Centro. Petrolina/PE. CEP: 56.304-917 E-mail: josaias.santana@facape.br

Wellington Dantas de Sousa Mestrado em Ciências Contábeis pela Fucape Bussines School - FUCAPE Professor no Instituto Federal Baiano - IFBAIANO Rua do Rouxinol, 115. Imbuí. Salvador/BA. CEP: 41.720-052 E-mail:wellington.sousa@ifbaiano.edu.br

\section{RESUMO}

Tendo como pano de fundo uma vinícola, localizada no Vale do São Francisco, que investe significativamente em pesquisa e desenvolvimento e, como tal, tem demonstrado importante continuidade no lançamento de novos produtos, o presente caso de ensino tem por objetivo discutir o processo de tomada de decisão estratégica no âmbito do desenvolvimento de produtos. Por meio de uma análise documental da aplicação da abordagem tradicional da técnica de Custeio-Alvo e, posteriormente, de um modelo modificado que incorpora o custo de oportunidade dos fundos investidos, 
Custeio-Alvo Incorporando o Custo de Capital para Tomada de Decisão: Um caso de Ensino João Carlos Hipólito Bernardes do Nascimento, Washington Juarez de Brito Filho, Juliana Reis

Bernardes, Josaias Santana dos Santos, Wellington Dantas de Sousa

conforme sugerido por Kee e Matherly (2006), Kee (2010) e Nascimento et al. (2013), os alunos terão a oportunidade de analisar a real viabilidade econômica do produto, pois não se mostrará, na prática, um processo tão intuitivo. O presente caso de ensino mostra-se útil para discussão nos cursos de graduação e de pós-graduação em Ciências Contábeis, Administração e Engenharia de Produção, nas disciplinas de Contabilidade de Custos, Contabilidade Gerencial e Análise de Custos.

Palavras-chave: Custo de capital. Tomada de decisão. Vinícolas. Custeio-Alvo.

\section{ABSTRACT}

Having as a background a winery, located in the Valley of the São Francisco, which invests significantly in research and development and, as such, has demonstrated important continuity in the launch of new products, this case study aims to discuss the strategic decision-making process in product development. Through a documentary analysis, the application of the traditional approach to goal calculation techniques and, subsequently, a modified model incorporating the opportunity cost of invested funds, as suggested by Kee and Matherly (2006), Kee (2010) and Nascimento et al. (2013), students will have the opportunity to analyze the real economic viability of the product, as it will not be shown in practice, such an intuitive process. The present teaching case is useful for discussion in the undergraduate and postgraduate courses in Accounting, Administration and Production Engineering, in the disciplines of Cost Accounting, Management Accounting and Cost Analysis.

Key words: Cost of capital. Decision making. Wineries. Target Costing.

\section{INTRODUÇÃO E CONTEXTUALIZAÇÃO DA SITUAÇÃO ESTUDADA}

A região do Vale do São Francisco (VFS), localizada na divisa entre os Estados de Pernambuco e Bahia, é atualmente um dos importantes centros produtores vinícolas do Brasil, apesar da baixa precipitação de chuva e da temperatura relativamente elevada (média de $27^{\circ}$, com máximas de $40^{\circ}$ ). Por meio de pesquisa e importação de tecnologias, sobretudo israelenses, o solo pobre e, até então, "pouco produtivo", transformou-se em uma grande "seara verde" (Zanini \& Rocha, 2010).

A vinícola Alpha, que, por questão de sigilo comercial, terá sua identidade preservada, atua nesse ramo há mais de 12 anos. Com foco na plantação da uva 
Custeio-Alvo Incorporando o Custo de Capital para Tomada de Decisão: Um caso de Ensino João Carlos Hipólito Bernardes do Nascimento, Washington Juarez de Brito Filho, Juliana Reis

Bernardes, Josaias Santana dos Santos, Wellington Dantas de Sousa

moscatel, a vinícola Alpha investe consideravelmente na Pesquisa e Desenvolvimento (P\&D) de novos produtos, sobretudo espumantes. Os produtores do VSF conseguem obter, atualmente, duas safras anuais, contra apenas uma dos demais polos produtores. Além dos produtos derivados da uva, algumas vinícolas têm investido no enoturismo.

Como o público desse tipo de produto é bastante exigente, a equipe de P\&D concentra grande parte dos recursos na identificação dos atributos/características valorizados pelo cliente, utilizando, para tal, o processo de análise de valor, buscando identificar os atributos esperados pelo consumidor.

Assim, além dos métodos tradicionais de custeio, a vinícola Alpha utiliza o Custeio-Alvo como técnica de gestão estratégica de custos no processo de tomada de decisão. Partindo da premissa de que o preço de venda reflete as especificações de produto valorizadas pelo cliente, o Custeio-Alvo é obtido pela diferença entre esse preço (valor máximo que o cliente se propõe pagar em decorrência dos atributos do produto) e a rentabilidade exigida pelo investidor. Atualmente, a vinícola Alpha detém todos os dados técnicos do produto $\mathrm{X}$, bem como sua demanda de mercado prevista. Porém, precisa decidir se o produto deve ou não ser lançado, utilizando-se, para tal, da técnica de Custeio-Alvo.

\section{TEXTO DO CASO}

Como apresentado anteriormente, a vinícola Alpha investe anualmente em P\&D, sobretudo na identificação dos atributos/características valorizados pelo cliente. Utiliza, para tal, o processo de análise de valor, buscando identificar os atributos esperados pelo consumidor. Nos últimos 12 meses, a equipe de P\&D parece ter conseguido desenvolver um espumante que apresenta um mix ótimo das características desejadas, sobretudo características organolépticas (perfume frutado), quantidade de açúcar e teor alcoólico. 
Custeio-Alvo Incorporando o Custo de Capital para Tomada de Decisão: Um caso de Ensino João Carlos Hipólito Bernardes do Nascimento, Washington Juarez de Brito Filho, Juliana Reis

Bernardes, Josaias Santana dos Santos, Wellington Dantas de Sousa

Tendo como insumo básico a uva moscatel produzida em duas safras anuais na própria sede, a vinícola Alpha realizou algumas pesquisas junto aos clientes com o objetivo de investigar se esses atributos, de fato, são valorizados pelo cliente e, sobretudo, o preço que esses consumidores estariam dispostos a pagar pelo produto que os possuísse. Com os resultados dessas pesquisas e os dados projetados de estrutura de custos e mercado do produto, a vinícola Alpha realizou o levantamento de dados, conforme Tabela 1:

Tabela 1

Informações da firma

\begin{tabular}{l|c}
\hline Preço unitário $(P)$ & 19,52 \\
\hline Vendas em unidades $(Q)$ & 98.000 \\
\hline Custo do Produto Vendido $(O)$ & 10,21 \\
\hline Investimento previsto $(I)$ & $3.232 .000,00$ \\
\hline Vida útil $(N)$ & 20 \\
\hline Margem requerida pelos investidores $(\alpha)$ & $20 \%$ \\
\hline Custo de Capital $(r)$ & $25 \%$ \\
\hline IR $(t)$ & $15 \%$
\end{tabular}

Nota. Fonte. Elaborada pelos autores

Muito embora as perspectivas sejam bastante otimistas, os acionistas só se dispõem a realizar o investimento caso seja garantida, antes do início da produção, a rentabilidade mínima exigida. Nesse aspecto, muito embora exista um mercado e o produto apresente os atributos desejados pelos clientes, questiona-se: 0 espumante $X$ deve ser lançado?

Com base na abordagem tradicional do Custeio-Alvo, é possível encontrar o Custo- Alvo projetado para o espumante X, conforme apresentado na Equação 1:

$$
\begin{gathered}
C A=P *(1-r) \quad(1) \\
C A=19,52 *(1-0,20) \\
C A=19,52 *(0,80) \\
\boldsymbol{C A}=\mathbf{1 5 , 6 2}
\end{gathered}
$$

Onde: 
Custeio-Alvo Incorporando o Custo de Capital para Tomada de Decisão: Um caso de Ensino João Carlos Hipólito Bernardes do Nascimento, Washington Juarez de Brito Filho, Juliana Reis Bernardes, Josaias Santana dos Santos, Wellington Dantas de Sousa

$\mathrm{CA}=$ Custo Alvo;

$\mathrm{P}=$ Preço unitário; $\mathrm{e}$

$r=$ Custo de Capital (margem requerida pelos investidores)

Como a vinícola Alpha trabalha com um custo estimado de produção de $\mathrm{R} \$$ 11,86 (CPV adicionado às despesas de depreciação), inferior ao custo obtido por meio da abordagem tradicional de Custeio-Alvo (valor de $R \$ 15,62$ ), a priori, a produção do produto deveria ser aceita conforme evidenciado na Tabela 2:

Tabela 2

Utilização do Custeio-Alvo tradicional

\begin{tabular}{l|c}
\hline $\mathrm{CPV}(\mathrm{O})$ & 10,21 \\
\hline$(+)$ Depreciação unitária (I/NQ) & 1,65 \\
\hline (=) Custo estimado produto (C) & $\mathbf{1 1 , 8 6}$ \\
\hline Custo-Alvo (equação 1) & 15,62 \\
\hline Resultado: & Aceito
\end{tabular}

Nota. Fonte. Elaborada pelos autores

É importante notar que, no cálculo efetuado via método tradicional, o custo de capital não foi considerado. Segundo Kee (2010), ao desconsiderar o custo marginal dos fundos investidos e superestimar o custo marginal de recursos de caixa relacionados à produção, o método tradicional de Custeio-Alvo pode acarretar a aceitação de produtos que apresentam Valor Presente Líquido (VPL) negativo, ao mesmo tempo que possibilita a rejeição de produtos que têm um VPL positivo. Assim, dada a sugestão de incorporação do custo dos fundos investidos no modelo tradicional de custeio, conforme sugerido por Kee e Matherly (2006), Kee (2010) e Nascimento et al. (2013), é importante considerar essa variável na análise do espumante X.

Assumindo que os preços dos produtos, custos unitários de produção, vendas anuais, tributação e custo de capital são constantes durante todo o ciclo de vida do produto, torna-se possível estimar o Custo-Alvo do produto por meio da Equação 2 (Kee, 2010). 
Custeio-Alvo Incorporando o Custo de Capital para Tomada de Decisão: Um caso de Ensino João Carlos Hipólito Bernardes do Nascimento, Washington Juarez de Brito Filho, Juliana Reis

$$
\begin{gathered}
C A=\frac{P Q(1-t) P V_{N, r}-I\left(1-\frac{P V_{N, r}}{N}\right)}{Q(1-t) P V_{N, r}} \\
C A=\frac{19,52 \times 98.000(1-0,15) \frac{1-(1,25)^{-20}}{0,25}-3.232 .000\left[1-\left(\frac{1-(1,25)^{-20}}{20 \times 0,25}\right]\right.}{98.000(1-0,15) \frac{1-(1,25)^{-20}}{0,25}} \\
C A=\frac{19,52 \times 98.000(0,85) \frac{1-0,011529215}{0,25}-3.232 .000\left[1-\left(\frac{1-0,011529215}{5}\right]\right.}{98.000(0,85) \frac{1-0,011529215}{0,25}} \\
C A=\frac{1.912 .960(0,85) \frac{0,988470785}{0,25}-3.232 .000\left[1-\left(\frac{0,988470785}{5}\right]\right.}{83.300 \frac{0,988470785}{0,25}} \\
C A=\frac{1.626 .016 * 3,953883140-3.232 .000[1-(0,197694157]}{83.300 * 3,953883140} \\
C A=\frac{6.429 .077,25-2.593 .052,48}{329.538,47} \\
329.538 .024,76
\end{gathered}
$$$$
C A=11,65
$$

Onde:

$\mathrm{CA}=$ Custo-Alvo;

$\mathrm{CPV}=$ Custo do Produto Vendido

$\mathrm{P}=$ Preço unitário; $\mathrm{e}$

$Q=$ vendas em unidades

$P Q=$ Preço unitário $x$ vendas em unidades

$\mathrm{PV}=$ present value (valor presente)

$\mathrm{N}=$ Vida útil

$r=$ Custo de Capital

$t=$ alíquota de tributação do Imposto de Renda 
Custeio-Alvo Incorporando o Custo de Capital para Tomada de Decisão: Um caso de Ensino João Carlos Hipólito Bernardes do Nascimento, Washington Juarez de Brito Filho, Juliana Reis

Bernardes, Josaias Santana dos Santos, Wellington Dantas de Sousa

Tabela 3

Informações e alíquotas

\begin{tabular}{l|c}
\hline Preço unitário $(P)$ & 19,52 \\
\hline Vendas em unidades $(Q)$ & 98.000 \\
\hline Custo do Produto Vendido $(O)$ & 10,21 \\
\hline Investimento previsto $(I)$ & $3.232 .000,00$ \\
\hline Vida útil $(N)$ & 20 \\
\hline Margem requerida pelos investidores $(\alpha)$ & $20 \%$ \\
\hline Custo de Capital $(r)$ & $25 \%$ \\
\hline IR $(t)$ & $15 \%$ \\
\hline
\end{tabular}

Nota. Fonte. Elaborada pelos autores

Nota-se que, após a incorporação do custo de capital ao custo alvo, tem-se o seguinte cenário de viabilidade, conforme Tabela 4:

Tabela 4

Custeio-Alvo após incorporação do custo de capital

\begin{tabular}{l|c}
\hline $\mathrm{CPV}(\mathrm{O})$ & 10,21 \\
\hline Depreciação unitária (I/NQ) & 1,65 \\
\hline Custo estimado produto (C) & $\mathbf{1 1 , 8 6}$ \\
\hline Custo Alvo (equação 2) & $\mathbf{1 1 , 6 5}$ \\
\hline Resultado: & Rejeitado \\
\hline No &
\end{tabular}

Nota. Fonte. Elaborada pelos autores

Nesse novo cenário, considerando o custo de capital, o custo estimado do produto mostrou-se superior ao Custo-Alvo, pelo que o espumante $\mathrm{X}$, inicialmente viável, não deveria ser lançado, tendo em vista que não se mostra economicamente viável. Calculando o Valor Presente Líquido (VPL) do produto após a incorporação do custo de capital, obtém-se a efetiva rentabilidade projetada do produto, conforme demonstrado na Equação 3 (Kee, 2010):

$V P L=\frac{(P-C) Q(1-t)\left[1-(1+r)^{-20}\right]}{r}-I\left(1-\frac{1-(1+r)^{-20}}{N r}\right)$

Assim, aplicando aos dados da empresa em análise, tem-se: 
Custeio-Alvo Incorporando o Custo de Capital para Tomada de Decisão: Um caso de Ensino João Carlos Hipólito Bernardes do Nascimento, Washington Juarez de Brito Filho, Juliana Reis

$$
\begin{gathered}
V P L=\frac{(19,52-11,86) * 98.000(1-0,15)\left[1-(1,25)^{-20}\right]}{0,25}-3.232 .000\left(1-\frac{1-(1,25)^{-20}}{20 \times 0,25}\right) \\
V P L=\frac{(7,66) * 98.000(0,85)[1-0,011529]}{0,25}-3.232 .000\left(1-\frac{0,9884717}{5}\right) \\
V P L=\frac{(7,66) * 83.300[0,988471]}{0,25}-3.232 .0000(1-0,197694) \\
V P L=\frac{630.721,48}{0,25}-3.232 .000(0,802305) \\
V P L=2.522 .885,85-2.593 .052,48 \\
V P L=(\mathbf{7 0 . 1 6 6 , 6 3 )}
\end{gathered}
$$

Pode-se notar que a incorporação do custo de capital no projeto leva a um VPL negativo, isto é, acarreta a alocação não eficiente de recursos financeiros. Assim, a vinícola Alpha não deve iniciar a execução do projeto nos termos apresentados, sendo necessária a reavaliação do processo produtivo, buscando-se a obtenção de redução de custos.

\subsection{Questão para discussão}

Dada essa realidade, a equipe de P\&D, ciente das potencialidades do produto e de sua importância para a vinícola, dado o atual contexto mercadológico, conseguiu remodelar o projeto por meio da identificação de novos materiais, substituição dos insumos atuais e estabelecimento de parcerias estratégicas com fornecedores, reduzindo o CPV (O) em 19,98\%.

Com esses resultados refletidos na planilha de custos, os acionistas foram convencidos a reduzirem o custo de oportunidade dos fundos investidos em $50 \%$. Nesse aspecto, a produção do espumante $\mathrm{X}$ deveria ser iniciada? 
Custeio-Alvo Incorporando o Custo de Capital para Tomada de Decisão: Um caso de Ensino João Carlos Hipólito Bernardes do Nascimento, Washington Juarez de Brito Filho, Juliana Reis

Bernardes, Josaias Santana dos Santos, Wellington Dantas de Sousa

\subsection{Glossário}

CPV: compreende o custo dos produtos vendidos (análogo ao CMV na Contabilidade Financeira).

Custo de Capital: taxa de remuneração exigida pelos investidores para financiar a atividade produtiva.

Depreciação: perda da capacidade de geração de caixa do ativo em decorrência da sua utilização.

Investimento: aplicação de recursos em determinada atividade produtiva.

Margem requerida: é a rentabilidade mínima desejada pelos investidores.

$P \& D=$ pesquisa e desenvolvimento.

Vida útil: é o período estimado para o qual, acredita-se, o produto (aí incluída a fábrica) possibilitará sua utilização com a plenitude de suas características fundamentais.

VPL: é o Valor Presente Líquido de uma série de prestações futuras (entradas e/ou saídas de caixa) descontadas a uma taxa de juros desejada, subtraído do desembolso inicial (investimento inicial) do fluxo de caixa. Em termos decisórios, o projeto só deve ser executado quando apresentar VPL positivo.

\section{NOTAS DE ENSINO}

Como apresentado anteriormente, este caso de ensino foi desenvolvido a partir dos insights propostos por Kee e Matherly (2006), Kee (2010) e Nascimento et al. (2013), mostrando-se extremamente relevante para discussão nos cursos de graduação e de pós-graduação em Ciências Contábeis, Administração e Engenharia de Produção, nas disciplinas de Contabilidade de Custos, Contabilidade Gerencial e Análise de Custos.

Roesch (2007) aponta que os casos para ensino na área de Administração são: 
Custeio-Alvo Incorporando o Custo de Capital para Tomada de Decisão: Um caso de Ensino João Carlos Hipólito Bernardes do Nascimento, Washington Juarez de Brito Filho, Juliana Reis

Bernardes, Josaias Santana dos Santos, Wellington Dantas de Sousa

"Relatos de situações da vida organizacional, construídos com propósitos educacionais específicos. Vêm sendo utilizados na formação gerencial há cerca de cem anos em universidades norte-americanas, e mais recentemente na Europa e na Ásia. No Brasil, são pouco usados, mas o interesse neste método de ensino-aprendizagem nas escolas de administração está ressurgindo, talvez pela proliferação de mestrados profissionais e MBAs."

Tendo em vista a interdisciplinaridade do presente caso de ensino, materializada na utilização de conceitos da Matemática Financeira e de Administração Financeira, é importante que esse caso seja aplicado em disciplinas que sejam ministradas em períodos simultâneos ou, especialmente, subsequentes a essas disciplinas.

É importante ressaltar que se trata de técnica contábil-financeira de elevada aplicabilidade prática e que admite enriquecimento paulatino dos exemplos considerados academicamente, aproximando-os da realidade profissional.

Após uma aula em que o tema 'Gestão Estratégica de Custos' e 'Custeio-Alvo' sejam apresentados e discutidos em sala de aula, o caso pode ser ministrado como atividade individual ou em grupo, para posterior correção e discussão em sala de aula.

Recomenda-se que a dinâmica da aula consista na apresentação dos pressupostos do caso em 10 minutos, antes que o caso seja posto à análise dos alunos. Prevê-se que os alunos tenham uma semana para efetuar suas análises e preparar suas conclusões. Espera-se que venham não só com a resposta à indagação posta como também com propostas para solucionar eventual situação desfavorável inicial. Ao final, um dos grupos ou indivíduo apresentará suas conclusões, franqueandose aos demais que intervenham no que puderem propor de forma diferenciada em relação ao exposto. Impõe-se que o moderador/professor incentive os alunos a refletirem sobre aspectos diferenciados do caso, fugindo do exame de apenas algumas das questões envolvidas. Sugerem-se debates em sala de, ao menos, uma hora.

O presente caso, acredita-se, tem o potencial de fomentar a reflexão, por parte dos discentes, acerca da iminente necessidade de incorporação do custo de oportunidade dos fundos investidos na técnica de Custeio-Alvo, tendo em vista que, em cenário contrário, sistematicamente, pode haver destruição do poder aquisitivo dos investidores, o que torna o investimento não atrativo. 
Custeio-Alvo Incorporando o Custo de Capital para Tomada de Decisão: Um caso de Ensino João Carlos Hipólito Bernardes do Nascimento, Washington Juarez de Brito Filho, Juliana Reis

Bernardes, Josaias Santana dos Santos, Wellington Dantas de Sousa

Secundariamente, o caso possibilita uma análise crítica da técnica de CusteioAlvo e, concomitantemente, da forma como ela é costumeiramente apresentada nas principais literaturas de Contabilidade de Custos, tendo em vista que não deixam clara a necessidade de incorporar o custo de oportunidade dos fundos investidos nos cálculos de Custeio-Alvo.

\section{RESPOSTA À QUESTÃO PROPOSTA}

Com a remodelagem dos valores inerentes à produção do espumante $X$ - isto é, redução do Custo do Produto Vendido (O) em 19,98\% e do custo de oportunidade dos fundos investidos em $50 \%$ - têm-se os seguintes dados (Tabela 5):

Tabela 5

Informações da firma após as reduções CPV e do custo de oportunidade dos fundos investidos

\begin{tabular}{l|c}
\hline Preço unitário $(P)$ & 19,52 \\
\hline Vendas em unidades $(Q)$ & 98.000 \\
\hline Custo do Produto Vendido $(O)$ & 8,17 \\
\hline Investimento previsto $(I)$ & $3.232 .000,00$ \\
\hline Vida útil $(N)$ & 20 \\
\hline Margem requerida pelos investidores $(\alpha)$ & $20 \%$ \\
\hline Custo de Capital $(r)$ & $12,50 \%$ \\
\hline IR $(t)$ & $15 \%$ \\
\hline
\end{tabular}

Nota. Fonte. Elaborada pelos autores

Utilizando a abordagem tradicional de Custeio-Alvo, o valor-Alvo mantém-se inalterado, isto é, Custeio-Alvo $=R \$ 15,62$. O mesmo sucede para a decisão de produzir, utilizando o modelo tradicional, conforme apresentado na Tabela 6:

Tabela 6

Custeio-Alvo utilizando a abordagem tradicional

\begin{tabular}{c|c}
\hline CPV $(\mathrm{O})$ & 8,17 \\
\hline Depreciação unitária (I/NQ) & 1,65 \\
\hline Custo estimado produto (C) & $\mathbf{9 , 8 2}$ \\
\hline Custo-Alvo (equação 1) & $\mathbf{1 5 , 6 2}$ \\
\hline Resultado: & Aceito
\end{tabular}

Nota. Fonte. Elaborada pelos autores 
Custeio-Alvo Incorporando o Custo de Capital para Tomada de Decisão: Um caso de Ensino João Carlos Hipólito Bernardes do Nascimento, Washington Juarez de Brito Filho, Juliana Reis Bernardes, Josaias Santana dos Santos, Wellington Dantas de Sousa

Mais uma vez assumindo que os preços dos produtos, custos unitários de produção, vendas anuais, tributação e custo de capital são constantes durante todo o ciclo de vida do produto, é estimado o Custo-Alvo do produto com a incorporação do custo de oportunidade dos fundos investidos por meio da Equação 4 (Kee, 2010):

$$
\left.\begin{array}{c}
C A=\frac{19,52 \times 98.000(1-0,15) \frac{1-(1,125)^{-20}}{0,125}-3.232 .000\left[1-\left(\frac{1-(1,125)^{-20}}{20 \times 0,125}\right]\right.}{98.000(1-0,15) \frac{1-(1,125)^{-20}}{0,125}} \\
C A=\frac{1.912 .960(1-0,15) \frac{0,905169170}{0,125}-3.232 .000\left[1-\left(\frac{1-0,094830830}{20 \times 0,125}\right]\right.}{83.300 \frac{1-0,094830830}{0,125}} \\
C A=\frac{1.912 .960(1-0,15) * 7,241353361-3.232 .000\left[1-\left(\frac{0,905169170}{2,50}\right]\right.}{83.300 \frac{0,905169170}{0,125}} \\
C A=\frac{1.626 .016,00 * 7,241353361-3.232 .000[1-(0,362067668]}{83.300 * 7,241353361} \\
C A=\frac{1.626 .016 * 7,241353361-2.061 .797,30}{603.204,73} \\
C A=\frac{1.626 .016 * 7,24135-2.061 .797,3}{603.204,73} \\
C A=\frac{9.712 .759,13}{603.204,73} \\
603.204,73
\end{array}\right]
$$$$
C A=16,10
$$ 
Custeio-Alvo Incorporando o Custo de Capital para Tomada de Decisão: Um caso de Ensino João Carlos Hipólito Bernardes do Nascimento, Washington Juarez de Brito Filho, Juliana Reis

Bernardes, Josaias Santana dos Santos, Wellington Dantas de Sousa

Agora, diferentemente do cenário anterior, mesmo após a incorporação do custo de capital, o espumante $X$ mostra-se economicamente viável, conforme a Tabela 7:

Tabela 7

Novo cálculo do Custeio-Alvo após incorporação do custo de capital

\begin{tabular}{l|c}
\hline CPV $(O)$ & 8,17 \\
\hline Depreciação unitária (I/NQ) & 1,65 \\
\hline Custo estimado produto (C) & $\mathbf{9 , 8 2}$ \\
\hline Custo-Alvo (equação 2) & $\mathbf{1 6 , 1 0}$ \\
\hline Resultado: & Aceito \\
\hline
\end{tabular}

Nota. Fonte. Elaborada pelos autores

Recalculando o VPL, conforme demonstrado na Equação 5 (Kee, 2010), têm-se:

$$
\begin{gathered}
V P L=\frac{(P-C) Q(1-t)\left[1-(1+r)^{-20}\right]}{r}-I\left(1-\frac{1-(1+r)^{-20}}{N r}\right) \\
V P L=\frac{(19,52-9,82) * 98.000(1-0,15)\left[1-(1,125)^{-20}\right]}{0,125} \\
-3.232 .000\left(1-\frac{1-(1,125)^{-20}}{20 \times 0,125}\right) \\
V P L=\frac{9,70 * 75.400,59}{0,125}-3.232 .000(0,637932332) \\
V P L=\frac{731.385,74}{0,125}-2.061 .797,30 \\
V P L=5.851 .085,92-2.061 .797,30 \\
V P L=\mathbf{3 . 7 8 9 . 2 8 8 , 6 2}
\end{gathered}
$$

Pode-se notar que, após a reformulação dos valores do projeto, mesmo após a incorporação do custo de capital, o projeto apresentou VPL positivo. Assim, a vinícola Alpha pode iniciar a execução do projeto nos termos apresentados.

É importante deixar claro que os alunos percebam quais são as variáveis que são endógenas (que dependem do desempenho da firma ou, pelo menos, podem ser 
por ela alteradas) e quais são exógenas, pois dependem do mercado. Assim, o lucro decorre da expectativa de rentabilidade do mercado de capitais para o segmento específico no qual a firma opera, dependendo da taxa de juros macroeconômica e da margem requerida. Ambas são, portanto, variáveis exógenas às decisões da vinícola. Já o Custeio-Alvo é uma variável endógena, tendo em vista que decorre do próprio desempenho da firma.

Ainda, importa que os alunos exercitem seu potencial analítico. Podem ser indagados se, de fato, é importante a incorporação do custo de oportunidade dos fundos investidos na técnica de Custeio-Alvo? Será que o mercado, especialmente em locais de menor movimentação de capitais, não é influenciado de alguma forma pelo desempenho da firma?

\section{REFERÊNCIAS}

Kee, R., \& Matherly, M. (2006). Decision control of products developed using target costing. Advances in Management Accounting, 15, 267-292.

Nascimento, J. C. H. B., Bernardes, J. R., Souza, W. D., \& Lancini, S. P. (2013). Custeio alvo, ferramenta estratégica de suporte às decisões de produção? Um estudo multi casos no Vale do São Francisco. CEP, Custos e @gronegócio Online, 9(3), 242269.

Roesch, S. M. A. (2007). Notas sobre a construção de casos para ensino. Revista de Administração Contemporânea, 11(2), 213-234.

Zanini, T. V., \& da Rocha, J. M. (2010). O Enoturismo no Brasil: um estudo comparativo entre as regiões vinícolas do Vale dos Vinhedos (RS) e do Vale do São Francisco (BA/PE). Revista Turismo em Análise, 21(1), 68-88.

Data de Submissão: 10/11/2017

Data de Aceite: 31/10/2018 